\title{
Performance comparison of classifiers on twitter sentimental analysis
}

\author{
Shruti Wadhwa1, Karuna Babber ${ }^{2}$, \\ ${ }^{\text {I} C h i e f ~ O p e r a t i n g ~ O f f i c e r, ~ N i d u s ~ T e c h n o l o g i e s ~ P v t . ~ L t d ., ~ C h a n d i g a r h, ~ I n d i a ~}$ \\ ${ }^{2}$ Assistant Professor, Panjab University, Chandigarh, India
}

\begin{abstract}
Twitter sentimental analysis is the way to examine polarity in tweeted opinions. The computational process involves implementing machine learning classifiers to categorize the tweets into positive, negative and neutral sentiments. To identify a suitable classifier for the task is a prime issue. In this paper we have presented the performance comparison of base classification techniques like Decision Tree, Random Forest, Naive Bayes, K-Nearest Neighbour and Logistic Regression on analysis of tweets. The results thus obtained show Logistic Regression analyze tweets with highest accuracy rate of $86.51 \%$ and the least performer comes out to be K-Nearest Neighbour with an average accuracy rate of $50.40 \%$.
\end{abstract}

Keywords: Twitter, sentimental analysis, machine learning, classifiers and algorithms 